\title{
Qualidade de vida e prevalência de dor na região cervical em acadêmicos
}

\author{
Quality of life and prevalence of pain in the cervical region in academics \\ Calidad de vida y prevalencia del dolor la región cervical em académicas
}

Tayane da Silva ${ }^{1 *}$, Arieliton Leal Oliveira ${ }^{1}$, Silmara Mendes dos Santos ${ }^{1}$, Tássio de Jesus Oliveira ${ }^{1}$, Lucas Sousa Guimarães ${ }^{1}$, Anáyra Macielly Rodrigues Ferreira ${ }^{1}$, Samia Daniele do Nascimento Ramos $^{1}$, Roberta Taizar Bezerra Silva Fontes ${ }^{1}$, Tamires Tomaz da Cruz ${ }^{1}$, Kelly Pereira Rodrigues dos Santos', Leonardo Carneiro Vieira ${ }^{1}$, Vanessa Moreira de Lima'1, Emigdio Nogueira Coutinho², Daniele Farias de Carvalho ${ }^{3}$, Cirley Pinheiro Ferreira ${ }^{4}$.

\section{RESUMO}

Objetivo: Avaliar a qualidade de vida e a prevalência de dor na região cervical em acadêmicos. Métodos: Trata-se de uma pesquisa de campo de delineamento transversal, descritiva com abordagem quantitativa, foram incluídos 289 adultos, com faixa etária de 18 a 50 anos de ambos os sexos, regularmente matriculadas em uma instituição de Ensino Superior. Foram aplicados os questionários SF36 e BECKPEI. Resultados: Dos entrevistados 157 (54,32\%) eram do sexo feminino e 132 (45,68\%) do sexo masculino, a dor na região cervical é prevalente em apenas 19,3\% dos indivíduos com frequência de 2 a 3 vezes na semana e com intensidade moderada, 62,28\% não praticam nenhum tipo de atividade física e passam de 0 a 1 hora por dia assistindo à televisão e até 5 horas no computador, quanto ao posicionamento para usar o computador, conversar ou estudar a mesa a maioria o faz de forma inadequada. Conclusão: A qualidade de vida, entre os jovens avaliados, foi considerada moderada e a dor cervical não foi a mais prevalente embora a maioria adotasse comportamentos sedentários e não praticasse atividade física.

Palavras-chave: Qualidade de vida, Cervicalgia, Estudantes.

\begin{abstract}
Objective: To evaluate the quality of life and prevalence of pain in the cervical region in academics. Methods: This is a cross-sectional, descriptive study with a quantitative approach. It will include young adults of both sexes, aged between 18 and 50 years, regularly enrolled in an institution of higher education, data were collected through the application of two SF36 and BECKPEI questionnaires. Results: Of the 189 individuals interviewed, 157 were female and 132 males aged 18 to 23 years, pain in the cervical region is prevalent in only $19.3 \%$ of individuals with frequency 2 to 3 times in the week and with moderate intensity, $62.28 \%$ do not practice any type of physical activity and spend from 0 to 1 hour a day watching television and up to 5 hours on the computer, regarding the positioning to use the computer, talk or study the table most does it improperly. Conclusion: Although cervical pain was not the most prevalent among the individuals interviewed, its prevalence was also significant, as the quality of life was considered moderate among the interviewees.
\end{abstract}

Key words: Quality of life, Cervical pain, Students.

\footnotetext{
${ }^{1}$ Centro Universitário de Ciência e Tecnologia do Maranhão (UNIFACEMA), Caxias-Ma.

*E-mail: Tayane_cx jk@hotmail.com

${ }^{2}$ Faculdade Santa Terezinha, São Luiz-MA.

3 Universidade Federal do Piauí (UFPI), Parnaíba-PI.

${ }^{4}$ Universidade Estadual do Piauí (UESPI), Teresina-PI.
} 


\section{RESUMEN}

Objetivo: Evaluar la calidad de vida y la prevalencia del dolor en la región cervical en académicos. Métodos: Este es un estudio descriptivo de corte transversal con un enfoque cuantitativo que incluirá adultos jóvenes de ambos sexos, de edades comprendidas entre 18 y 50 años, inscritos regularmente en una institución de educación superior, datos se recogieron mediante la aplicación de dos cuestionarios SF36 y BECKPEI. Resultados: De los 189 individuos entrevistados, 157 eran mujeres y 132 hombres de 18 a 23 años, el dolor en la región cervical es prevalente en solo el 19.3\% de los individuos con frecuencia de 2 a 3 veces en la semana y con intensidad moderada, el $62.28 \%$ no practica ningún tipo de actividad física y pasa de 0 a 1 hora al día mirando televisión y hasta 5 horas en la computadora, con respecto a la posición para usar la computadora, hablar o estudiar la mesa más lo hace incorrectamente Conclusión: aunque el dolor cervical no fue el más frecuente entre las personas entrevistadas, su prevalencia también fue significativa, ya que la calidad de vida se consideró moderada entre los entrevistados.

Palabras clave: Calidad de vida, Cervicalgia, Estudiantes.

\section{INTRODUÇÃO}

O acúmulo de tarefas exigidas aos universitários no decorrer da graduação e a quantidade de tempo destinada aos estudos são motivos que, na maioria das vezes, os levam a adotarem maus hábitos posturais e alimentares, não praticarem atividade física orientada, podendo até chegar a um estado de sobrepeso, aumentando assim a probabilidade de ocorrer disfunções musculoesqueléticas (GUEDES FG, MACHADO APNB, 2008). Borges RG (2011) afirma que a incidência e prevalência de dores nas costas já foi experimentada pela população em algum momento da vida. O termo "dor nas costas" é um termo bastante utilizado relacionados as disfunções musculoesqueléticas, mais especificamente para designar as algias na coluna vertebral (IGUTI AM, BASTOS TF, BARROS ABA, 2015).

A cervicalgia ou dor cervical é definida como a presença de dor na região posterior do pescoço, superior das escápulas ou na zona dorsal alta (OVERBECK G, 2015). Esta condição raramente se inicia de maneira súbita, em geral pode estar relacionada com movimentos bruscos, longa permanência em posição forçada, esforço ou trauma (SOBRAL ALP et al. 2010). Na população adulta jovem em geral, a incidência de disfunções na região cervical é de $12 \%$ a $34 \%$, ocorrendo em alguma fase da vida, com possível recorrência da dor nos cinco anos seguintes. A coluna cervical é a parte da coluna que apresenta um alto grau de mobilidade e pouca estabilidade, sendo assim mais susceptível a lesões. Dessa forma, a disfunção nessa região é uma condição comum que afeta boa parte da população, representada por um conjunto de sinais e sintomas que envolvem dor, limitação da amplitude dos movimentos fisiológicos, alteração da sensibilidade e/ou dor à palpação dos músculos cervicais (FERREIRA ACT, MARTINI FAN, PIRES PF, 2013).

A disfunção que afeta a região cervical pode causar um grande desconforto devido à dor e a incapacidade podendo afetar as atividades acadêmicas e as atividades de vida diárias (KANCHANOMAI S et al., 2011). Esta vem gerando um impacto significativo na saúde da população em geral e está comumente associada a qualidade de vida, influenciando diretamente na redução de seus indicadores. Acredita-se que cerca de $70 \%$ a $85 \%$ da população mundial está susceptível a desenvolver quadros agudos ou subagudos de dor em qualquer região das costas. No Brasil chega a ser um dos principais problema de saúde. A literatura mostra que a dor pode comprometer a rotina de vida diária dos indivíduos com incapacidade temporariamente ou permanentemente (FERREIRA GD et al., 2011; SANTOS LRCS, 2014).

A realização do presente estudo se dá pelo grande número de queixas apresentadas nos consultórios médicos e de fisioterapia relacionadas às cervicalgias e diminuição da capacidade funcional afetando diretamente a qualidade de vida dos acadêmicos. Diante do exposto, o objetivo geral deste estudo foi avaliar a qualidade de vida e a prevalência de dor na região cervical em acadêmicos, de cinco cursos distintos, onde foram avaliados a qualidade de vida através dos questionários Medical Outcomes Study 36-Item Short-Form Health Survey (SF-36) e a dor na coluna, correlacionando a prevalência dessa dor cervical com à qualidade de vida, por meio do questionário Back Pain and Body Posture Evaluation Instrument (BackPEI). 


\section{MÉTODOS}

O presente estudo trata-se de uma pesquisa de campo de delineamento transversal, descritiva com abordagem quantitativa. A pesquisa foi realizada em uma faculdade privada do interior do Maranhão que conta com um total de 3.527 alunos em 15 cursos de graduação. A amostra escolhida para a aplicação dos instrumentos foi a comunidade acadêmica composta de 289 pessoas. Esta amostra foi definida com base no cálculo de amostragem para população finita, com grau de confiança de 95\% e margem de erro de 5\%.

Os acadêmicos foram selecionados de forma aleatória. Conforme a declaração positiva para a participação da pesquisa, os acadêmicos receberam o Termo de Consentimento Livre e Esclarecido (TCLE) e tiveram um prazo máximo de 2 dias para análise e devolução dele, confirmando sua decisão sobre a participação no estudo. Foram incluídos e participaram da pesquisa adultos com idade entre 18 a 50 anos, de ambos os sexos, regularmente matriculadas em uma instituição Privada de Ensino Superior e que pertencessem aos cursos de Fisioterapia, Educação Física, Arquitetura, Engenharia Civil e Análise e Desenvolvimento de Sistemas (ADS). Foram excluídos da pesquisa os acadêmicos que apresentaram algum tipo patologia instalada na coluna e que não atendiam aos critérios de inclusão supracitados.

Foi realizado a estatística descritiva (frequência) para os dados referentes à caracterização do perfil dos indivíduos avaliados quanto a faixa etária, sexo e curso utilizando-se o programa IBM SPSS 21 (IBM SPSS $21,2012)$. Para respostas do tipo sim ou não sem agrupamentos, utilizou-se teste de Qui-quadrado $(P<0,05)$ através do Programa de Linguagem de Frequência (PROC FREQ) do programa estatística SAS (SAS INSTITUTE, 2001). Para elaboração dos gráficos foi utilizado o software Sigma plot versão 10.0 (SYSTAT SOFTWARE Inc, 2006).

Este projeto de pesquisa respeitou os aspectos éticos que envolvem os estudos desta natureza, conforme a resolução 466/2012 do Conselho Nacional de Saúde, que institui as normas de pesquisa em saúde. Foi submetido ao Comitê de Ética do Centro Universitário de Ciências e Tecnologia do Maranhão (UniFacema) e aprovado com o número de CAAE: 91806318.3.0000.8007.

\section{RESULTADOS}

A população do estudo foi composta por 289 indivíduos com idades entre 18 e 50 anos, sendo $54,32 \%$ (157) do sexo feminino e 45,68\% (132) do sexo masculino, estando estes enquadrados em sua maioria na faixa etária de 18 a 23 anos, representando $57,44 \%$ da amostra. Quanto aos cursos avaliados $27,68 \%(80)$ pertenciam ao curso de Fisioterapia, 27,68\% (80) a Engenharia Civil, 19,38\% (56) a Arquitetura, 12,80\% (37) a Educação Física e 12,46\% (36) ao curso de Análise e Desenvolvimento de Sistema (Tabela 1).

Tabela 1 - Caracterização do perfil dos indivíduos avaliados quanto ao curso, sexo e faixa etária.

\begin{tabular}{cccc}
\hline \multicolumn{2}{c}{ Parâmetros } & Frequência & $\%$ \\
\hline Faixa etária & $18-23$ & 166 & 57,44 \\
& $23-28$ & 97 & 33,56 \\
& $28-33$ & 17 & 5,88 \\
& $33-38$ & 7 & 2,43 \\
& $38-43$ & 2 & 0,69 \\
\hline Sexo & Feminino & 157 & 54,32 \\
& Masculino & 132 & 45,68 \\
\hline Curso & ADS & 36 & 12,46 \\
& Arquitetura & 56 & 19,38 \\
& Educação Física & 37 & 12,80 \\
& Engenharia Civil & 80 & 27,68 \\
& Fisioterapia & 80 & 27,68 \\
\hline
\end{tabular}

Legenda: Análise e Desenvolvimento de Sistemas (ADS). Fonte: Silva T, et al. 2019. 
No que diz respeito à região da coluna com maior incidência de dor, a lombar foi a mais prevalente, com maior frequência entre os acadêmicos do curso de Educação Física $(70,97 \%)$. A segunda região da coluna com maior prevalência de dor foi a cervical, com destaque para o curso de Arquitetura (26,53\%), seguido dos cursos de Engenharia Civil (25,64\%), Fisioterapia (22,78\%), Educação Física (19,35\%) e ADS (14,81\%). Quando questionados sobre a frequência de dor na região da coluna (no intervalo dos últimos três meses), a maioria dos avaliados responderam sentir dor de 3 a 4 vezes por semana, com prevalência entre os acadêmicos de Engenharia Civil que representando $35,5 \%$ dos pesquisados (Tabela 2).

Tabela 2 - Incidência e frequência de dor.

\begin{tabular}{|c|c|c|c|c|c|c|}
\hline \multirow{2}{*}{\multicolumn{3}{|c|}{ Cursos }} & \multicolumn{4}{|c|}{ Regiões da Coluna (\%) } \\
\hline & & & Cervical & Lombar & Torácica & Sacral \\
\hline \multicolumn{3}{|c|}{ ADS } & 14,81 & 62,97 & 11,11 & 0 \\
\hline \multicolumn{3}{|c|}{ Arquitetura } & 26,53 & 57,14 & 8,16 & 2,04 \\
\hline \multicolumn{3}{|c|}{ Educação Física } & 19,35 & 70,97 & 6,45 & 3,23 \\
\hline \multicolumn{3}{|c|}{ Engenharia Civil } & 25,64 & 50 & 19,23 & 0 \\
\hline \multicolumn{3}{|c|}{ Fisioterapia } & 22,78 & 48,1 & 21,52 & 1,27 \\
\hline $\begin{array}{c}\text { Frequência de } \\
\text { dor }\end{array}$ & ADS & Arquitetura & Educação Física & \multicolumn{2}{|c|}{ Engenharia Civil } & Fisioterapia \\
\hline 1 vez por mês & 14,81 & 14,29 & 9,68 & \multicolumn{2}{|c|}{2,56} & 3,8 \\
\hline 1 vez por semana & 14,81 & 22,45 & 22,58 & \multicolumn{2}{|c|}{20,51} & 20,25 \\
\hline 2 a 3 vezes & 29,64 & 26,53 & 22,58 & \multicolumn{2}{|c|}{35,9} & 32,91 \\
\hline 4 ou mais vezes & 7,41 & 12,24 & 19,35 & \multicolumn{2}{|c|}{17,95} & 20,25 \\
\hline 1 única vez & 11,11 & 10,2 & 16,13 & \multicolumn{2}{|c|}{12,82} & 8,87 \\
\hline Não sabe & 22,22 & 14,29 & 9,68 & \multicolumn{2}{|c|}{10,26} & 13,92 \\
\hline
\end{tabular}

Legenda: Análise e Desenvolvimento de Sistemas (ADS). Fonte: Silva T, et al. 2019.

Quanto à intensidade de dor, conforme escala visual analógica de dor (EVA), a maioria dos participantes relataram dor de grau 4. Houve maior prevalência entre os acadêmicos do curso de Arquitetura $(30,61 \%)$, seguido de ADS (29,63\%), Engenharia Civil (24,36\%), Fisioterapia (18,99\%) e Educação Física (16,13\%) (Gráfico 1).

Gráfico 1 - Intensidade da dor nos últimos 3 meses.

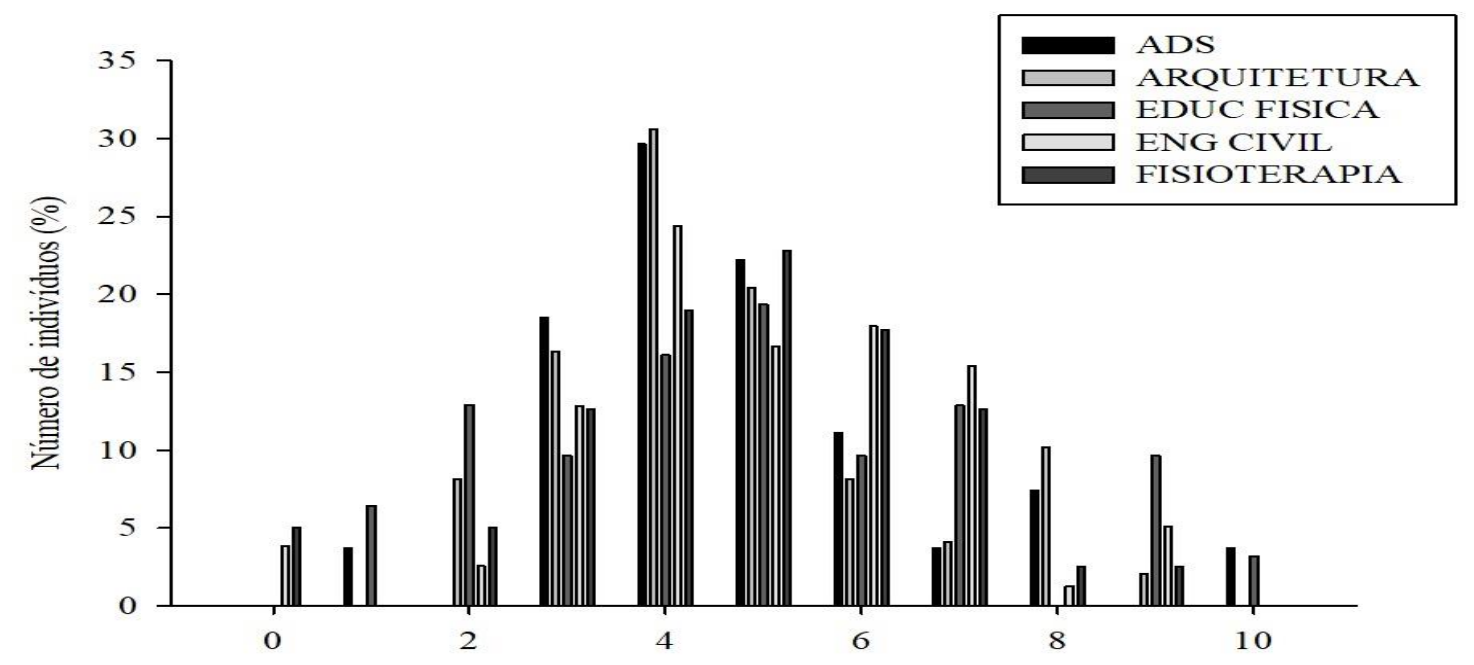

Legenda: Análise e Desenvolvimento de Sistemas (ADS). Fonte: Silva T, et al. 2019. 
Quando questionados sobre a práticas de exercícios físicos, a maioria dos acadêmicos entrevistados responderam não praticar nenhum tipo de exercício físico, representando um percentual de 62,28\%. Este dado foi significativamente maior $(P<0,05)$ daqueles que realizam algum tipo de atividade física.

No momento em que os entrevistados foram questionados sobre o tempo de permanência sentado assistindo à televisão, observou-se que a maioria passava cerca de 0 a $1 \mathrm{~h}$ por dia assistindo TV, com destaque para o curso de Educação Física (67,57\%). Já aqueles que assistiam $6 \mathrm{~h}$ ou mais eram em sua maioria do curso de Engenharia Civil (11,25\%), conforme gráfico 2A. Em relação ao tempo de permanência na posição sentada utilizando o computador, os indivíduos que cursam ADS (16,67\%) foram os mais prevalentes com duração de 6 horas ou mais. Seguido de Engenharia Civil $(38,75 \%)$ com exposição de 4 a 5 h e o curso de Arquitetura (28,57\%) com 2 a 3 h (Gráfico 2B).

Gráfico 2 - Tempo de permanência sentado em frente à TV $(A)$, Tempo de permanência sentado em frente ao computador (B).
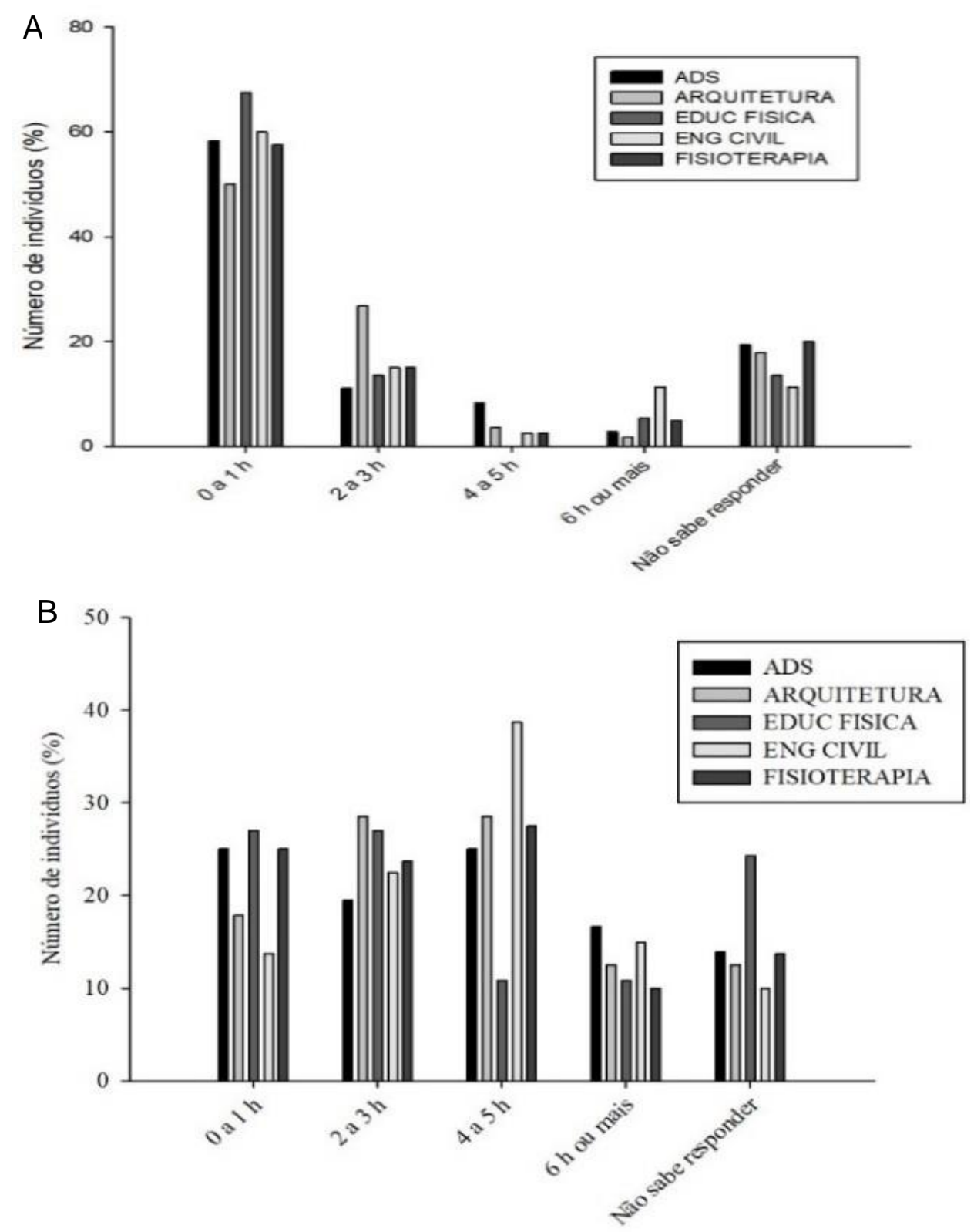

Legenda: Análise e Desenvolvimento de Sistemas (ADS). Fonte: Silva T, et al. 2019. 
Foi observado que a maioria dos entrevistados se sentavam de forma errada durante o uso do computador. A má postura se mantinha quando sentados em uma cadeira ou banco para conversar (Gráfico 3A) e estudar à mesa (Gráfico 3B).

Gráfico 3 - A Posição para sentar-se em cadeira ou banco ao conversar; B- Posição para estudar na mesa.

A
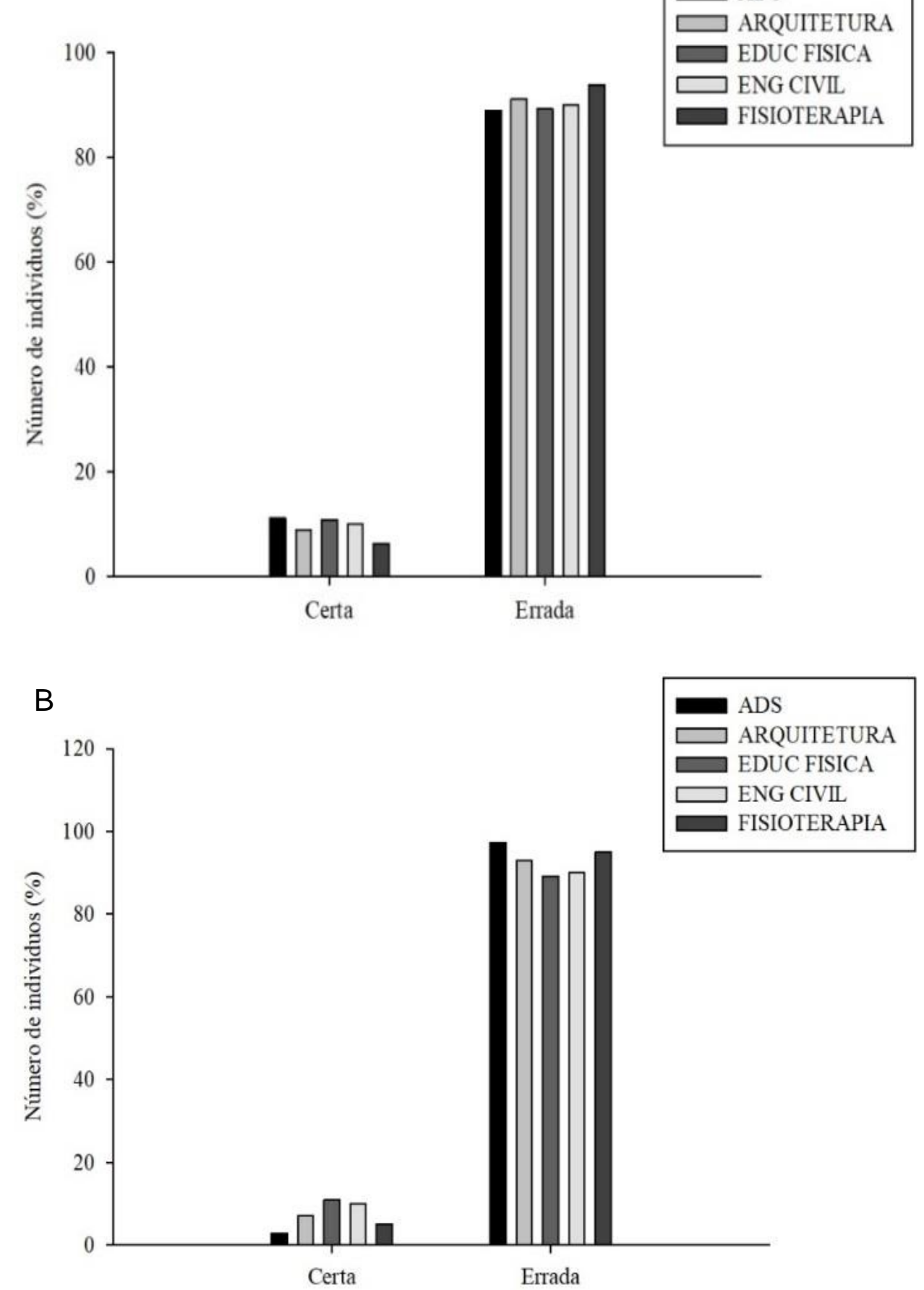

Legenda: Análise e Desenvolvimento de Sistemas. (ADS). Fonte: Silva T, et al. 2019.

Embora haja uma grande prevalência de dor na região da coluna, tanto lombar quanto cervical, essa dor não interferiu significativamente na realização das atividades de vida diárias desses indivíduos (Gráfico 4). 
Gráfico 4 - A dor impediu as atividade de vida diária (AVD's).

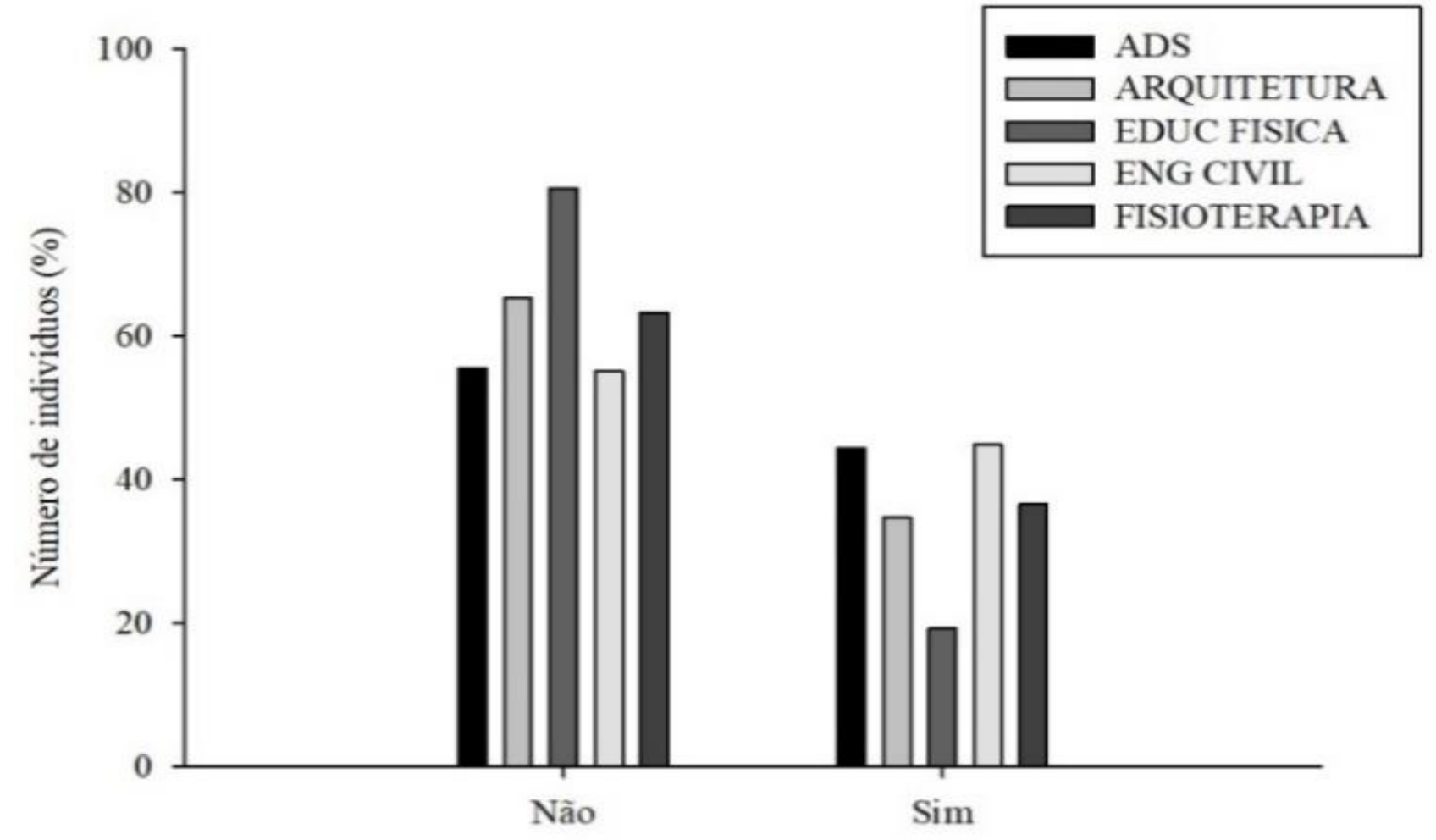

Legenda: Análise e Desenvolvimento de Sistemas (ADS). Fonte: Silva T, et al. 2019.

\section{DISCUSSÃO}

Os dados do estudo demonstraram que a maioria dos indivíduos entrevistados são do sexo feminino. Este fato se dá por uma maior inserção da mulher no contexto do ensino superior e pela maior proporção de mulheres demograficamente falando, com faixa etária de 18 a 23 anos (APOSTULOS JLA et al., 2011).

Neste estudo todos os cursos relataram sentir dor nos últimos três meses com frequência de 2 a 3 vezes na semana, a região de prevalência da dor foi a região lombar cerca de $50 \%$, o que já era esperado devido à alta prevalência de dor nessa região em diversos estudos como e o caso do estudo de Vey APZ, Silva AC e Lima FST (2016) que investigaram a relação entre as horas em que os alunos de graduação passam na posição sentada e a queixa de dor nas costas, onde $47,9 \%$ dos indivíduos apresentaram dor na região lombar e somente $19,3 \%$ apresentam dor na cervical.

Com relação a frequência da dor Sendrez AJ (2015) corroboram em partes com o presente estudo, pois dos 228 dos escolares pesquisados $25,5 \%$ das meninas relataram sentir dor de 2 a 3 vezes na semana e apenas $21,4 \%$ dos meninos relataram um único episódio de dor, tais diferenças se dão pela forma de divisão estatísticas escolhidas por ambos os estudos.

Entretanto, a região cervical também teve um grande percentual de queixas, ficando em segundo lugar sendo referida por cerca de $30 \%$, dos indivíduos, o mesmo foi encontrado no estudo de Depintor JDP et al, (2016) realizado em São Paulo com 826 participantes, onde a prevalência de dor na região cervical foi de $12,5 \%$ dos indivíduos, ficando também em segundo lugar quando comparado à região lombar.

Com relação à intensidade da dor cervical, os entrevistados, em sua grande maioria, referiram sentir dor grau 4-5 segundo a escala visual analógica de dor (EVA), o que é considerado um nível moderado de dor, uma boa parte dos indivíduos referiram também sentir dor grau 6 e 7, beirando o nível severo, estando em consonância com o estudo epidemiológico de Ferreira CF e Rosa LH (2008) onde 13 indivíduos, cerca de 
$87 \%$ da população pesquisada sentem dor de nível moderado e 2 indivíduos, $13 \%$ da população referiram sentir dor severa.

Os resultados mostraram que a maioria dos acadêmicos (62,28\%) não praticava nenhum tipo de exercício ou atividade física, o que pode está diretamente ligada com alta prevalência de dor uma vez que o sedentarismo e considerado um dos fatores de risco para o aparecimento de quadros álgicos na região da coluna, e embora $37,72 \%$ praticavam algum exercício a maioria não soube responder com que frequência praticava exercícios. Esses dados são divergentes ao estudo realizado por Vey APZ, Silva AC e Lima FST (2013) onde $57,77 \%$ dos entrevistados praticavam exercícios físicos.

Ao analisar o tempo em que os acadêmicos assistem à televisão e usam o computador, constatou-se que a maior parcela dos participantes do estudo permanece de 0 a 1 hora por dia assistindo à televisão sendo 0 curso de Educação Física foi o mais prevalente com $67,57 \%$, já em relação ao uso do computador a maioria relatou permanecer em um período de até cinco horas por dia, no computador. Esses dados podem ser comparados ao estudo de Vitta A et al. (2011), com escolares do ensino fundamental de $5^{\circ}$ a $8^{\circ}$ serie da cidade de Bauru, onde destacou que $73 \%$ e $67,1 \%$ dos escolares assistiam à televisão por um período superior a duas horas diárias e usam o computador por um período de até duas horas diárias, respectivamente. Contudo, no presente estudo não houve associação significativa entre o tempo de permanência assistindo TV ou horas por dia no computador, com a prevalência de dor na região cervical.

Ao analisar a posição sentado em algumas atividades como estudar a mesa, conversar ou usar o computador observou-se que a maioria dos indivíduos apresentaram a forma inadequada de sentar-se para tais atividades, do mesmo modo Sedrez JA et al, (2015) em seu estudo com crianças e adolescentes de 7 a 18 anos, constatou que os mesmo, também tinham o habito de sentar para conversar, usar o computador e estudar a mesa de forma errônea.

O que segundo Pompeu KD e Freitas CLR (2009) podem gerar forças nocivas a coluna ocasionando alterações significativas na coluna. Em relação à análise multivariável, o presente estudo encontrou associação significativa entre a forma de sentar-se (para estudar a mesa, usar o computador e conversar) com a prevalência de dor na região cervical e lombar.

Sá CLL et al, (2015) em uma pesquisa de cunho exploratório com escolares do $5^{\circ}$ ano do ensino fundamental verificou-se que mais da metade dos indivíduos que relataram sentir dor nas costas, não encontraram nenhum impedimento na realização das atividades de vida diária, somente $33,3 \%$ dos avaliados reportaram que a dor impede a realização de atividades. Resultados semelhantes foram encontrados no presente estudo onde a maioria dos avaliados alegam não encontrar impedimentos na realização das atividades por motivos de dor. Com relação a pequena parcela da população que referiram algum tipo de impedimento das atividades o curso de engenharia civil foi o que mais encontrou impedimentos com 44,87\%, seguidos dos cursos de ADS (44,44\%), fisioterapia (36,71\%), Arquitetura (34,69\%) e educação física $(19,35 \%)$. Contudo a análise multivariável do presente estudo não encontrou associação significativa entre a presença de dor e a qualidade de vida.

\section{CONCLUSÃO}

A grande maioria dos fatores investigados pela pesquisa tiveram um alto nível de significância, tais fatores podem estar diretamente relacionados à presença de dor na região cervical uma vez que sua prevalência foi a segunda mais significativa, ficando atrás apenas da dor lombar. E quanto a qualidade de vida, não foi encontrado resultados relevantes que comprovem que a dor na região da coluna cervical possa interferir nos indicadores da qualidade de vida. Tendo em vista a alta prevalência de dor na coluna, tanto cervical quanto lombar, faz-se necessário a adoção de estratégias para a prevenção e tratamento desses indivíduos, a fisioterapia é a ciência da saúde que estuda, previne e trata os distúrbios cinéticos funcionais, o profissional fisioterapeuta tem propriedades suficiente para atuar em intervenções educativas sobre maus hábitos posturais e auxiliar na redução de quadros de dores musculoesqueléticas, sendo considerado um profissional de extrema importância no processo de reabilitação e prevenção da saúde. 


\section{REFERÊNCIAS}

1. APÓSTOLO JLA, et al. Depressão, ansiedade e estresse em usuários de cuidados primários de saúde. Revista Latino-Americana de Enfermagem, v. 19, n. 2, p. 348-353, 2011.

2. BORGES RG, et al. Efeitos da participação em um Grupo de Coluna sobre as dores musculoesqueléticas, qualidade de vida e funcionalidade dos usuários de uma Unidade Básica de Saúde de Porto Alegre-Brasil. Motriz: Revista de Educacao Fisica, v. 17, n. 4, p. 719-727, 2011.

3. DEPINTOR JDP, et al. Prevalence of chronic spinal pain and identification of associated factors in a sample of the population of São Paulo, Brazil: cross-sectional study. Sao Paulo Medical Journal, v. 134, n. 5, p. 375-384, 2016.

4. FERREIRA ACT, et al. Comparação da Amplitude do Movimento Cervical em Mulheres com Disfunção Cervical e Assintomáticas. Saúde em Revista, v. 13, n. 33, p. 31-37. 2013.

5. FERREIRA CF, ROSA LH. Estudo epidemiológico sobre os fatores de risco das algias de coluna vertebral. http://www.wgate.com.br/conteudo/medicinaesaude/fisioterapia/reumato/algias_fatoresri sco. htm em, v. 20, n. 10, p. 09, 2008.

6. FERREIRA GD, et al. Prevalência de dor nas costas e fatores associados em adultos do Sul do Brasil: estudo de base. 2011.

7. FONTELLES MJ, et al. Metodologia da pesquisa científica: diretrizes para a elaboração de um protocolo de pesquisa. Revista Paraense de Medicina, v. 23, n. 3, p. 1-8, 2009.

8. GUEDES FG, MACHADO APNB. Fatores que influenciam no aparecimento das dores na coluna vertebral de acadêmicos de fisioterapia. Estação Científica Online [Periódico online], v. 5, p. 1-10, 2008.

9. IGUTI AM, et al. Dor nas costas em população adulta: estudo de base populacional em Campinas, São Paulo, Brasil. Cad. Saúde Pública, Rio de Janeiro, v. 31, n. 12, p. 2546-2558, Dec. 2015.

10. KANCHANOMAI S, at al. Risk factors for the onset and persistence of neck pain in undergraduate students: 1 -year prospective cohort study. BMC public health, v. 11, n. 1, p. 566, 2011.

11. OVERBECK G. Cervicalgia crônica na relação do questionário de qualidade de vida sf-36 e a classificação internacional de funcionalidade, incapacidade e saúde. 2015. Monografia (Fisioterapia), 28f., Universidade de Santa cruz do Sul, Santa Cruz do Sul, 2015.

12. POMPEU KD, FREITAS CLR. A influência da Educação Física sobre a postura dinâmica sentada de escolares. Rev. Digital, v. 10, n.2, 2009.

13. SÁ CL, et al. Avaliação do peso e da forma de carregar o material escolar de alunos que deixam seus livros na escola. Saúde (Santa Maria), v. 41, n. 1, p. 105-110, 2015.

14. SANTOS LRCS. Dor nas costas: prevalência e fatores associados em comunidades quilombolas da Bahia. 2014. Tese (Saúde Pública), 111f, Faculdade de medicina da Universidade Estadual de Minas gerais, Belo Horizonte, MG, 2014.

15. SEDREZ JA, et al. Fatores de risco associados a alterações posturais estruturais da coluna vertebral em crianças e adolescentes. Revista Paulista de Pediatria, v. 33, n. 1, p. 72-81, 2015.

16. SOBRAL MLP, et al. Estudo da prevalência de algias na coluna vertebral em residentes de cirurgia cardiovascular: estudo inicial. Rev Bras Med Trab, v. 11, n. 2, p. 82-9, 2013.

17. VEY APZ, et al. Análise de dor nas costas em estudantes de graduação. Disciplinarum Scientia| Saúde, v. 14, n. 2, p. 217-225, 2016.

18. VITTA A, et al. Prevalence of lower back pain and associated factors in students. Cadernos de saude publica, v. 27 , n. 8, p. 1520-1528, 2011. 